\title{
Edema Limbs
}

National Cancer Institute

\section{Source}

National Cancer Institute. Edema Limbs. NCI Thesaurus. Code C78262.

Swelling due to an excessive accumulation of fluid in the upper or lower extremities. 